\title{
Dual-color Multiresponsive Rhodamine Derivative and its Photochromic Enhancement Behavior in Polyurethane
}

\author{
Guoping Zeng ${ }^{1, a}$, Hengfeng $\mathrm{Li}^{2, \mathrm{~b}}$, Jun Zhang ${ }^{1, \mathrm{c}}$, Fu-qian Sun ${ }^{1, \mathrm{~d}}$, Yaqing Weng ${ }^{1, \mathrm{e}}$, \\ Shuang Wang ${ }^{1, \mathrm{f}}$ \\ ${ }^{1}$ Institute of Applied Chemistry, Jiangxi Academy of Sciences, Nanchang, 330096, Jiangxi, China \\ ${ }^{2}$ School of Materials Science and Engineering, Central South University, Changsha, 410083, \\ Hunan, China \\ aemail:nczgp@126.com; bemail: lihf@csu.edu.cn; 'email: 814057519@qq.com; \\ demail: sunfuqian@126.com; eemail: 40378569@qq.com; 'email: 406834464@qq.com
}

Keywords: Dual-color; Multiresponsive; Rhodamine; Polyurethane; Photochromic

\begin{abstract}
In this study, a rhodamine derivative (RH-PY) with dual color (blue green to orange) and multiple response (light, acid gas, and force) was synthesized. The photochromic enhancement behavior of rhodamine derivative in polyurethane matrix was explored. By complexing polyurethane with RH-PY, the open-form lifetime of RH-PY can extend from 3 days to more than one month. To the best of our knowledge, this is the longest open-form lifetime of rhodamine derivatives reported so far. Rhodamine with long open-form lifetime has great potential in developing outstanding photochromic materials. This method is simple and inexpensive, and non-covalent preparation suits for more rhodamine derivatives.
\end{abstract}

\section{Introduction}

Environment-sensitive materials are becoming increasingly attractive owing to their potential applications in molecular switches,[1-4] logic gates,[5] optical data storage,[6] optoelectronic devices,[7] controllable drug delivery,[8] etc. Compared to the monotone sensitive type, the environment-sensitive materials have been developed to various sensitive types including light, electricity, force, gas, magnet, and chemical compounds.[9-11] One approach for obtaining this type of material is by introducing some certain intelligent organic molecules. Among the reported intelligent molecules, rhodamine is an outstanding molecule owing to its long-wavelength absorption and emission, high absorption coefficient and quantum efficiency, and good photostability.[12] The closed-form of rhodamine derivatives can undergo structural transformation to the open-form by the stimulations of light, force, $\mathrm{pH}$, and chemical compounds.[13] Its wide applications spread to diverse areas including industrial coloration, biomarkers, and fluorometric probes.[14, 15] However, the introduction of rhodamine derivatives as one type of environment-sensitive material is still challenging. One problem is the fluorescence emissions of their closed-forms. Another critical problem is the photochromic behavior of rhodamine, rhodamine derivatives as photochromic molecules are very scarce in literature because of the short lifetime of their open-form.[12] The longest lifetime photochromic behavior of rhodamine derivatives is reported by B. Tang, where the lifetime of the open-form was extended to several hours. However, this promotion depended on the ion complex and the specific structural design, thus the scope of application narrowed to some certain rhodamine derivatives. Herein, we report a novel approach for dual-color multi-responsive rhodamine derivative RH-PY as shown in Scheme 1. By combining pyrene with rhodamine, RH-PY can present excellent photochromic behavior. 


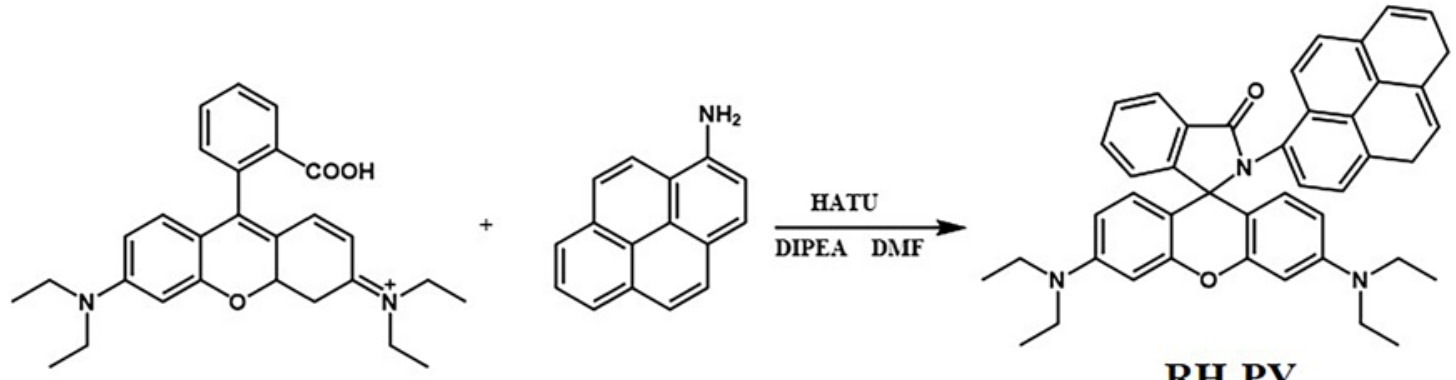

RH-PY

Scheme 1. Synthesis of RH-PY

\section{Experimental}

\section{Materials and characterization.}

Rhodamine B, 1-aminopyrene, N,N-Diisopropylethylamine (DIPEA) and O-(7-Azobenzotriazol -1-yl)- 1,1,3,3-tetramethyluronium hexafluorophosphate (HATU) were purchased from Aladdin and used without further purification. Fluorescence spectroscopic studies were performed on a fluorescence spectrophotometer (Horiba Jobin Yvon FluoroMax-4 NIR, NJ, USA). Absorbance spectroscopic studies were performed using a spectrometer (Cintra 20, GBC, Australia).

\section{Synthesis of RH-PY.}

Rhodamine B (300 mg, $0.68 \mathrm{mmol}$ ) and HATU (418 mg, $1.36 \mathrm{mmol}$ ) were dissolved in DMF (8 $\mathrm{mL})$. To that solution, DIPEA $(0.48 \mathrm{~mL}, 2.7 \mathrm{mmol})$ was added dropwise and reacted at room temperature for $30 \mathrm{~min}$, followed by adding a solution of 1-aminopyrene (176.5 $\mathrm{mg}, 0.81 \mathrm{mmol})$ in DMF (1mL). The resulting reaction mixture was stirred at room temperature for $12 \mathrm{~h}$. DMF was then evaporated under vacuum, and the residue was poured in distilled water to get red precipitate. The precipitates thus obtained was collected and further purified by silica gel column chromatography with ethyl acetate: $\mathrm{DCM}=1: 20$, affording RH-PY as a pink powder (yield 70\%). ${ }^{1} \mathrm{H}$ NMR (400 MHz, $\left.\mathrm{CDCl}_{3}\right) \delta 8.18(\mathrm{~d}, \mathrm{~J}=7.5 \mathrm{~Hz}, 1 \mathrm{H}), 8.08-8.00(\mathrm{~m}, 1 \mathrm{H}), 7.99-7.92(\mathrm{~m}$, 2H), 7.88 (d, J = 8.2 Hz, 2H), 7.76 (d, J = 9.3 Hz, 1H), 7.69 (t, J = 5.5 Hz, 1H), 7.64 (d, J = 9.3 Hz, 2H), 7.43 (d, J = 7.4 Hz, 1H), 6.86 (dd, J = 20.1, 8.7 Hz, 1H), $6.51(\mathrm{~d}, \mathrm{~J}=7.7 \mathrm{~Hz}, 3 \mathrm{H}), 6.30$ (d, J = $8.5 \mathrm{~Hz}, 1 \mathrm{H}), 6.23$ (s, 2H), 5.77 (s, 1H), 3.42 (dt, J = 13.7, 7.0 Hz, 4H), 3.11 (q, J = 6.8 Hz, 4H), 1.24 $(\mathrm{t}, \mathrm{J}=6.9 \mathrm{~Hz}, 6 \mathrm{H}), 0.92(\mathrm{t}, \mathrm{J}=6.9 \mathrm{~Hz}, 6 \mathrm{H})$. ESI-TOF: $\mathrm{m} / \mathrm{z}$ calcd 642.4 for $\mathrm{C}_{44} \mathrm{H}_{41} \mathrm{~N}_{3} \mathrm{O}_{2}, 642.32$; found, $642.4\left(\mathrm{M}+\mathrm{H}^{+}\right)$.

\section{Results and Discussion}

\section{Synthesis of RH-PY.}

Traditional way of synthesizing sensitive rhodamine starts with rhodamine B, amino compounds, in the presence of weak base.[16] However, for RH-PY, the stearic hindrance of pyrene slows the reactivity, and the reaction does not go to completion. Therefore, HATU, DIPEA were used to activate the carboxyl group of rhodamine $\mathrm{B}$, the target product can be obtained with relative high yield (70\%).

\section{Acid gas-chromic performance of RH-PY.}

Rhodamine is well known for its $\mathrm{pH}$ sensitive properties. It is highly sensitive to acid in the solution-state, enabling its wide application as $\mathrm{pH}$-dependent probes.[17] In acidic solutions, the ring-opened form is predominant, Although this acid-sensitive equilibrium has been well known in solutions, its properties in the solid states are not well examined. Therefore, relative test of acid gas-sensitive property of RH-PY was proposed in this study.

Figure 1(A) shows the test device. Concentrated $\mathrm{HNO}_{3}$ acid was placed in a bottle up to $1 / 3$ volume. The sample was cast on a glass and then set on the bottleneck to fully cover the bottleneck. Concentrated $\mathrm{HNO}_{3}$ is highly volatile, as expected; RH-PY is highly sensitive to gaseous $\mathrm{HNO}_{3}$. As shown in Figure 1(B), the absorption of initial sample is centered at $350 \mathrm{~nm}$. Upon exposure to fuming concentrated $\mathrm{HNO}_{3}$ vapor, a new peak centered at $570 \mathrm{~nm}$ began to appear. This peak is 
assigned to the open-form of RH-PY, demonstrating that the ring-opening occurred upon the stimulation of gaseous $\mathrm{HNO}_{3}$. The acid gas response of RH-PY was accomplished in 5 min.

(A)

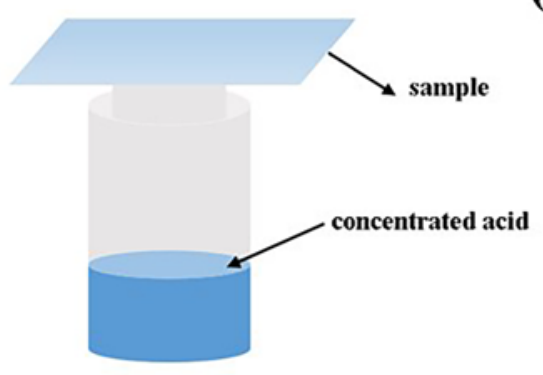

(B)

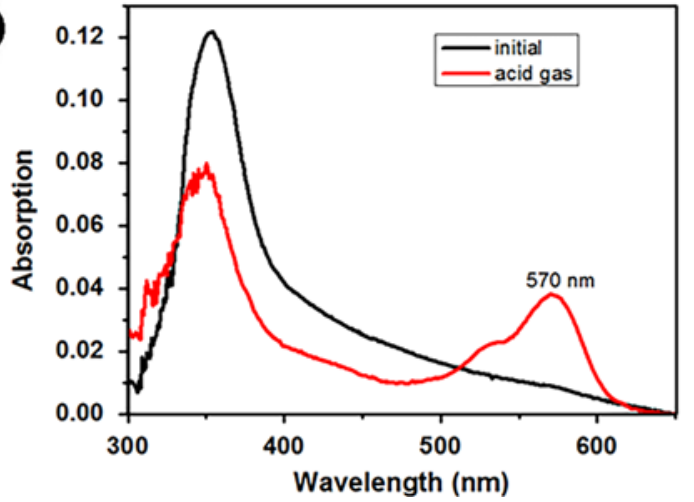

Figure 1. (A) Device schematic of acid-gas sensitive test (B); Absorption variation of RH-PY under the stimuli of acid gas.

\section{Mechanochromic performance of RH-PY.}

RH-PY showed reversible color switch from bright blue to orange under UV and room light by grinding. Upon heating the sample, the orange color was fully recovered to the initial state. The variation in the emission spectra upon grinding can be $170 \mathrm{~nm}$. Such mechanochromic properties with large emission variation, arose from the excimer emission of pyrene unit and the chemical reaction of rhodamine unit from the spirolactam form to the ring-opened amide, significantly differs from the previously reported mechanochromic materials showing two color variations, and the mechanisms of control the molecular structures or molecular orientation separately. To the best of our knowledge, this is a rare example of the color change by a single molecule, attributing to the arrangement of chromophores and the mechanochemical reaction.

To evaluate the mechanochromic properties of RH-PY, relative optical spectra measurements were performed. RH-PY, in its initial state, shows a sharp peak at $350 \mathrm{~nm}$ in the absorption spectra, and $450 \mathrm{~nm}$ in the fluorescence spectra respectively. Upon grinding, a new peak at $570 \mathrm{~nm}$ in the absorption spectra, and $586 \mathrm{~nm}$ in fluorescence spectra began to appear and gradually increased with the force added. By heating at $70^{\circ} \mathrm{C}$ for several minutes, new peaks (570 nm in the absorption spectra, $586 \mathrm{~nm}$ in the fluorescence spectra) fully restored to the initial state (Figure 2). The peak at $570 \mathrm{~nm}$ was assigned to the open form of rhodamine derivative, and the mechanochromism is attributed to the ring-opening of RH-PY. The large variation in the optical spectra $(220 \mathrm{~nm}$ in the absorption spectra, $136 \mathrm{~nm}$ in the fluorescence spectra) allowed easy detection.
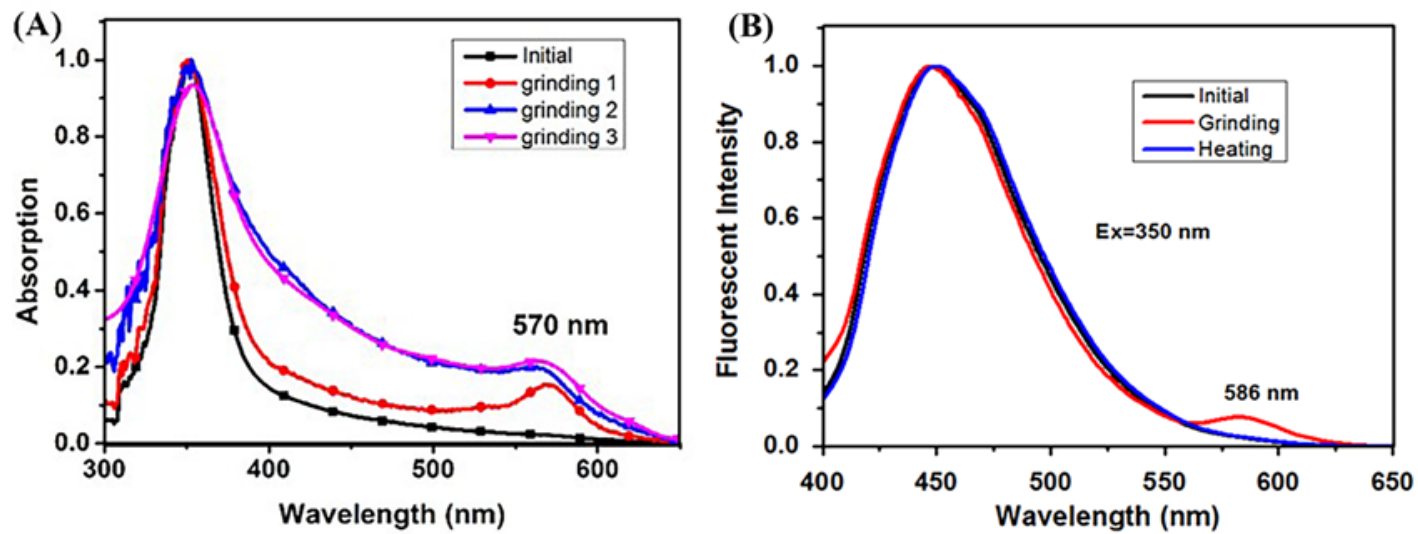

Figure 2. (A) Absorbance and (B) Fluorescence spectra of RH-PY fluorophore powder with and

\section{Photochromic performance of RH-PY.} without grinding.

The photochromic property of RH-PY was evaluated in dichloromethane (DCM) solution. Upon irradiation by UV light (365 nm), a new peak in the near-infrared region evolved and increased gradually with time. As shown in Figure 3, RH-PY initially exhibits a single peak centered at 350 $\mathrm{nm}$, and within $3 \mathrm{~min}$ irradiation of UV light, a new peak centered at $560 \mathrm{~nm}$ evolves. With increasing irradiation time, the intensity of new peak increases gradually. This new peak is 
attributed to the open-form of RH-PY, demonstrating that the ring opening occurred in this period. After 20 min irradiation, this increment could not be obviously observed, as the ring-opening conversion rate reached saturation. Then, upon the storage in the dark, the intensity of this new peak began to decline gradually. Specially, the rate of decrease of the intensity of RH-PY is slower, and this new peak did not disappear until storage for 3 days. Compared to the reported photochromic rhodamine derivatives with only several hours open-form lifetime, RH-PY shows outstanding photochromic property, with the open-form lifetime of 3 days.
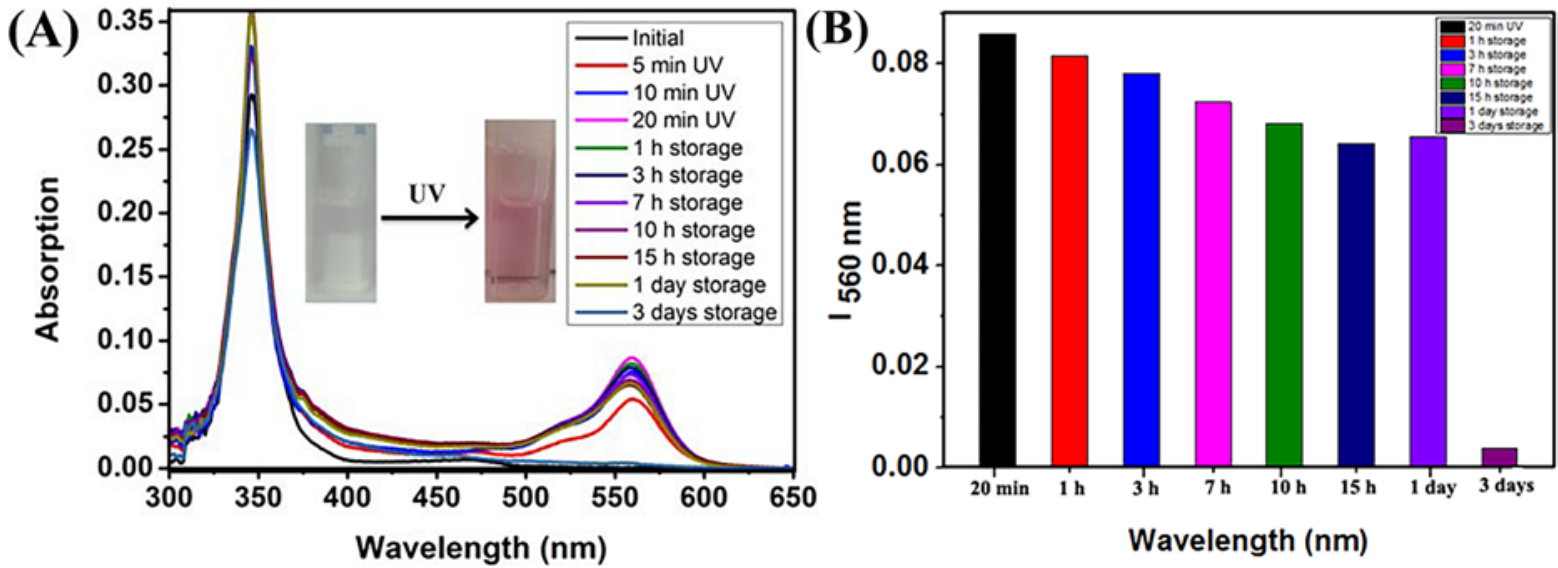

Figure 3. (A) Variation in the absorption spectrum of RH-PY under UV irradiation and dark storage. (B) Collection of $\mathrm{I}_{560}$ in the absorption spectrum of RH-PY under UV irradiation and dark storage.

\section{Photochromic enhancement behavior of RH-PY in polyurethane.}

The photochromism of RH-PY/PU complex was first investigated in DCM solution with a concentration of $1 \times 10^{-5} \mathrm{M}$ ). As shown in Figure 4, the new peak centered at $560 \mathrm{~nm}$ was obviously observed after 50 min UV irradiation and then gradually increased with increasing irradiation time. $\mathrm{RH}-\mathrm{PY} / \mathrm{PU}$ complex in DCM solution responded slower than RH-PY in DCM solution, requiring 50 min to show any obvious photochromic performance. After 50 min UV irradiation RH-PY/PU coating complex was then stored in the dark. Interestingly, this new peak did not disappear until 6 days' storage in the dark. Compared to the isolated RH-PY in DCM solution with same concentration. RH-PY/PU complex in DCM solution responded slowly to the light, but its open-form lifetime was highly extended from 2 days to 6 days. This enhancement can be used to develop photochromic rhodamine derivatives in various application areas.
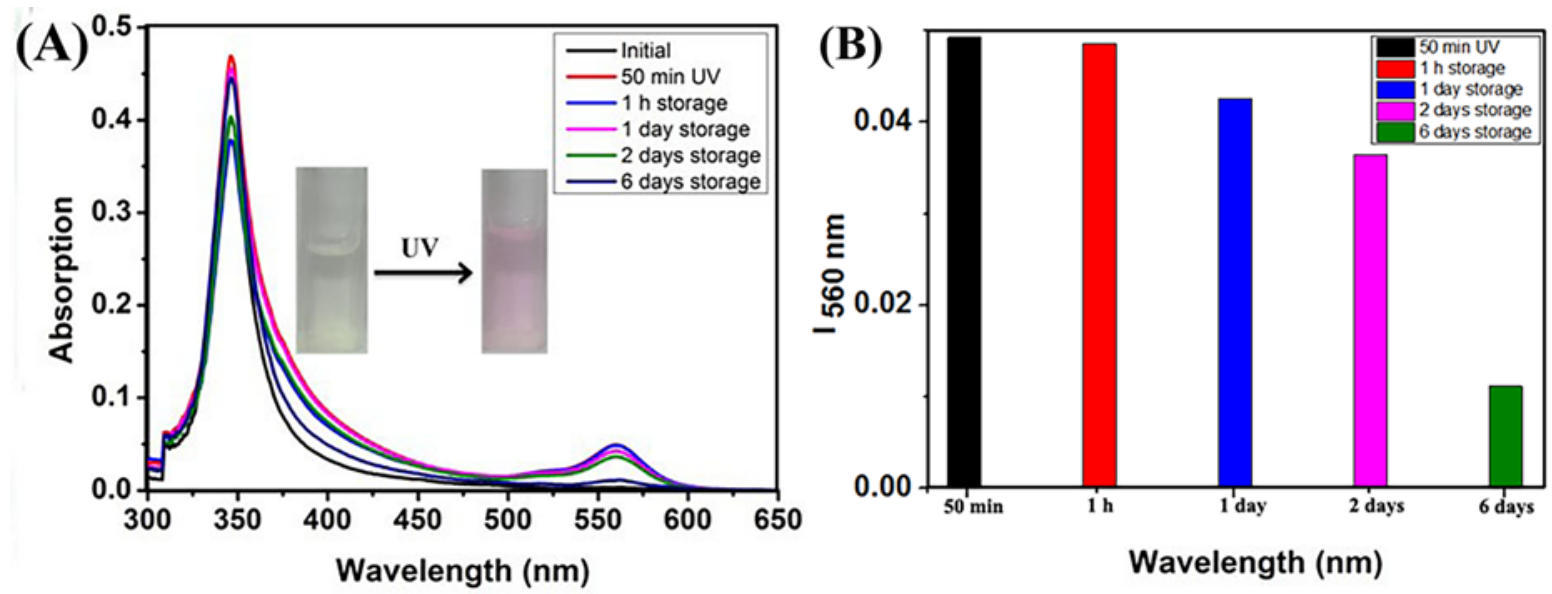

Figure 4. (A) Variation in the absorption spectrum of RH-PY/PU complex in DCM solution under UV irradiation and dark storage. (B) Collection of $\mathrm{I}_{560}$ in the absorption spectrum of RH-PY/PU coating complex in DCM solution under UV irradiation and dark storage.

Interestingly, the RH-PY/PU complex also showed photochromism in the solid state, which cannot be realized by isolated RH-PY. Moreover, the photochromic performance of RH-PY/PU complex in the solid state is far more outstanding than either RH-PY/PU complex in solution or 
isolated RH-PY solution. The RH-PY/PU film was obtained by casting method, its photochromic performance is shown in Figure 5. Upon UV irradiation for $30 \mathrm{~min}$, a new peak centered at $560 \mathrm{~nm}$ evolved obviously. Then the film was stored in the dark to investigate its open-form lifetime. The RH-PY/PU film shows extremely long open-form lifetime and did not show any decrease within 10 days. Therefore, by complexing RH-PY with polyurethane, the open-form lifetime can be extended to more than 1 month, promote more than $93 \%$ of isolated RH-PY. Such significant improvement endows rhodamine with outstanding photochromic performance, thus increasing its application area.
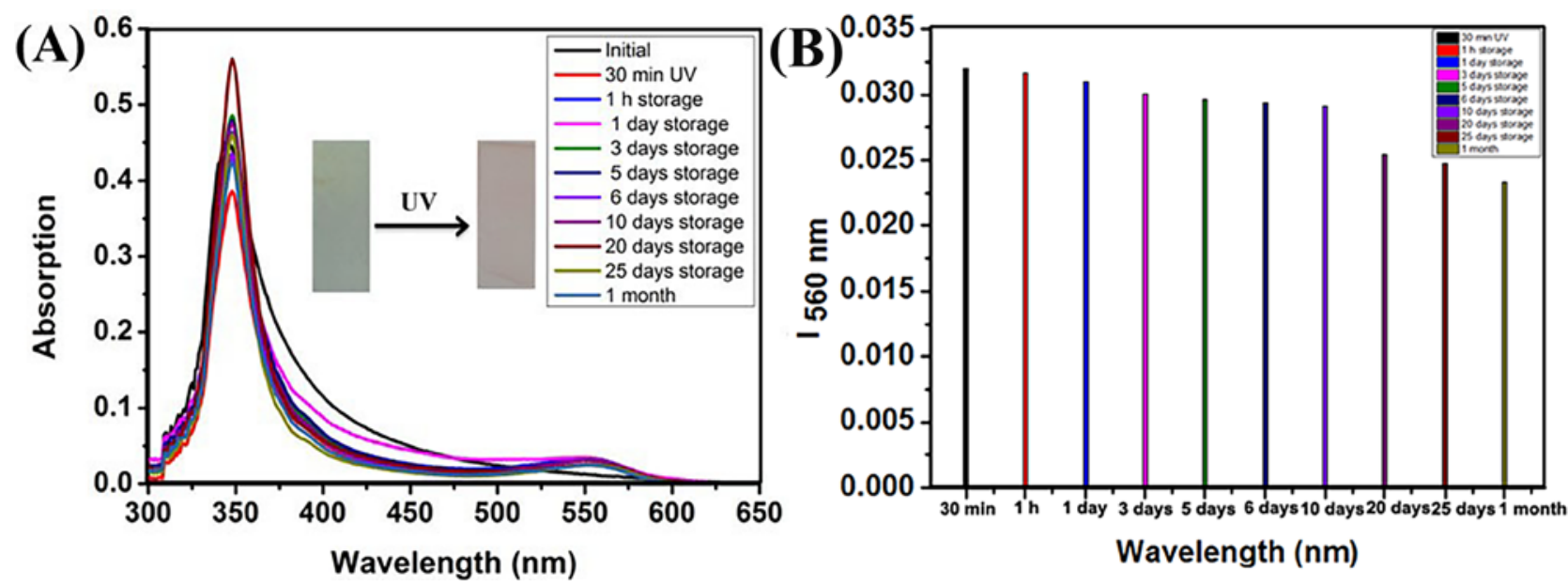

Figure 5. (A) Variation in the absorption spectrum of RH-PY/PU complex in solid state under UV irradiation and dark storage. (B) Collection of $\mathrm{I}_{560}$ in the absorption spectrum of RH-PY/PU coating complex in solid state under the UV irradiation and dark storage.

To investigate the mechanism of the enhancement behavior of polyurethane, the chemical structure of polyurethane was first analyzed. the main chemical structure of polyurethane consists of numerous $\mathrm{N}-\mathrm{H}$ bonds and $\mathrm{C}=\mathrm{O}$ bonds that can induce strong hydrogen-bonding interactions. Hydrogen-bonding interaction plays a crucial role in photochromism of some sensitive molecules. For example, the hydrogen bonding of cyclodextrin has been utilized to extend the open-form lifetime of spiropyran derivatives.[18] Therefore, we speculated that the hydrogen bonding of polyurethane also functions in enhancing the photochromic performance of RH-PY.

\section{Conclusions}

A rhodamine derivative RH-PY with dual-color and multiresponsive switches was prepared. This sensitive molecule responded to various stimuli including acid gas, force, and UV light. Triggered by these stimuli, the fluorescence of RH-PY changed from blue-green to orange. Specially, RH-PY possesses outstanding photochromic performance. Compared to the traditional photochromic rhodamine derivatives with several hours open-form lifetime, the lifetime of the open form of RH-PY was 2 days. Furthermore, a novel approach for extending the open-form lifetime of rhodamine derivatives was explored. By physical complexing polyurethane with RH-PY, the open-form lifetime of RH-PY after UV irradiation extended to more than one month. This way is simple and convenient, and the non-covalent preparation enables this method suitable for various rhodamine derivatives. By introducing this method, more potential rhodamine derivatives could show enhanced photochromism with extremely long open-form lifetime.

\section{Acknowledgement}

In this paper, the research was sponsored by the External Cooperation Program of Jiangxi Academy of Sciences (2014-07), the External Cooperation Program of Jiangxi Academy of Sciences (2016-YCXY-02). the Key Research Plan of Jiangxi Province (2016IBBE50097), and the Key Invention Patent Industrialization Plan of Jiangxi Province (20161BBM26035). 


\section{References}

[1] Davis D. A., Hamilton A., Yang J. L., et al., Force-induced activation of covalent bonds in mechanoresponsive polymeric materials[J]. Nature, 2009, 459(7243): 68-72.

[2] Wagner K., Byrne R., Zanoni M., et al., A Multiswitchable Poly(terthiophene) Bearing a Spiropyran Functionality: Understanding Photo- and Electrochemical Control[J]. J Am Chem Soc, 2011, 133(14): 5453-5462.

[3] Shao N., Jin J. Y., Wang H., et al., Design of Bis-spiropyran Ligands as Dipolar Molecule Receptors and Application to in Vivo Glutathione Fluorescent Probes[J]. J Am Chem Soc, 2010, 132(2): 725-736.

[4] Achilleos D. S., Hatton T. A., Vamvakaki M., Light-Regulated Supramolecular Engineering of Polymeric Nanocapsules[J]. J Am Chem Soc, 2012, 134(13): 5726-5729.

[5] Raymo F. M., Alvarado R. J., Giordani S., et al., Memory effects based on intermolecular photoinduced proton transfer[J]. J Am Chem Soc, 2003, 125(8): 2361-2364.

[6] Kawata S., Kawata Y., Three-dimensional optical data storage using photochromic materials[J]. Chem Rev, 2000, 100(5): 1777-1788.

[7] Zacharias P., Gather M. C., Kohnen A., et al., Photoprogrammable Organic Light-Emitting Diodes[J]. Angew Chem Int Ed, 2009, 48(22): 4038-4041.

[8] Chen Z. W., Zhou L., Bing W., et al., Light Controlled Reversible Inversion of Nanophosphor-Stabilized Pickering Emulsions for Biphasic Enantioselective Biocatalysis[J]. J Am Chem Soc, 2014, 136(20): 7498-7504.

[9] Goswami S., Das A. K., Maity A. K., et al., Visual and near IR (NIR) fluorescence detection of Cr3+ in aqueous media via spirobenzopyran ring opening with application in logic gate and bio-imaging[J]. Dalton Transactions, 2014, 43(1): 231-239.

[10]Nam Y. S., Yoo I., Yarimaga O., et al., Photochromic spiropyran-embedded PDMS for highly sensitive and tunable optochemical gas sensing[J]. Chem Commun, 2014, 50(32): 4251-4254.

[11] Ciardelli F., Ruggeri G., Pucci A., Dye-containing polymers: methods for preparation of mechanochromic materials[J]. Chem Soc Rev, 2013, 42(3): 857-870.

[12]Li K., Xiang Y., Wang X. Y., et al., Reversible Photochromic System Based on Rhodamine B Salicylaldehyde Hydrazone Metal Complex[J]. J Am Chem Soc, 2014, 136(4): 1643-1649.

[13] Sahana A., Banerjee A., Lohar S., et al., Rhodamine-Based Fluorescent Probe for Al3+ through Time-Dependent PET-CHEF-FRET Processes and Its Cell Staining Application[J]. Inorg Chem, 2013, 52(7): 3627-3633.

[14]Shi W., Ma H. M., Spectroscopic probes with changeable pi-conjugated systems[J]. Chem Commun, 2012, 48(70): 8732-8744.

[15]Yang Y. J., Seidlits S. K., Adams M. M., et al., A Highly Selective Low-Background Fluorescent Imaging Agent for Nitric Oxide[J]. J Am Chem Soc, 2010, 132(38): 13114-13116.

[16]Li Y. H., Zhao Y. R., Chan W. H., et al., Selective Tracking of Lysosomal Cu2+ Ions Using Simultaneous Target- and Location-Activated Fluorescent Nanoprobes[J]. Anal Chem, 2015, 87(22): 11599-11599.

[17]Lv H. S., Huang S. Y., Zhao B. X., et al., A new rhodamine B-based lysosomal pH fluorescent indicator[J]. Anal Chim Acta, 2013, 788: 177-182.

[18]Zhang S. X., Fan M. G., Liu Y. Y., et al., Inclusion complex of spironaphthoxazine with gamma-cyclodextrin and its photochromism study[J]. Langmuir, 2007, 23(18): 9443-9446. 